\title{
Study on Construction Technology of Concrete Structure in Con- struction Engineering
}

\author{
Bozhou Key Engineering Administration Bureau of Bozhou City, Anhui Province 236800
}

\section{Construction concrete structure}

The concrete structure of the concrete structure is mainly composed of reinforced concrete structure, prestressed concrete structure, and plain concrete structure. The main material is composed of concrete and some related auxiliary materials. This is a new structure. As the main use of concrete in the construction industry, the concrete structure itself has the following characteristics: First, in the concrete structure of the process of making relatively simple, easy to operate, the technical requirements are relatively low; Is the production of concrete structure of the relatively rich raw materials, its quality and cheap; Moreover, the concrete structure of its own fire resistance and durability is relatively good, concrete structure and other brick structure shock resistance and storm performance are better, That is to say that it can be very good to adapt to the phenomenon of various types of natural disasters; the next concrete structure is a good overall and plasticity, so to a large extent also convenient after the use of its performance is also Easy to adjust, can be fully applied to all kinds of construction projects; the last is the concrete structure can effectively use all kinds of industrial waste, such as coal ash, slime and slag, and so on such waste.

2 The construction of concrete structure of the construction technology analysis

2.1 Material control and preparation
First, aggregate quality control. The aggregate used in the construction of concrete structures is mainly sand and gravel. The amount is mainly affected by the amount of concrete structure. However, the choice of aggregate will directly affect the quality of concrete structure construction. Second, it is the quality of cement. Cement types can be divided into general water, special cement and special cement, which are used in the construction of concrete structures used in the application of materials, so you have to be based on the actual situation to optimize the application of cement and performance. Strict guarantee of the quality of cement can guarantee the scientific and rational allocation of concrete structure, in the actual construction process, to do the cement grade, variety and factory batch number one by one clear, and the other storage of cement warehouse have to keep dry, to prevent the occurrence of cement damp situation. When the concrete structure is arranged, the raw materials such as gravel, cement and water are stirred and fused together according to the corresponding proportions, and the raw materials are arranged by means of adaptation and experiment. Can be more in line with the corresponding technical standards.

\subsection{Concrete structure pouring technology}

The first is to clean up the construction site debris in a timely manner. In the concrete structure before pouring, it is necessary to carry out a comprehen

\begin{abstract}
In order to analyze the application of construction technology concrete construction technology in order to maximize the quality of construction, it is necessary to grasp the basic operation steps of each stage of construction. Scientific and reasonable concrete structure construction technology, not only can effectively protect the entire building construction concrete structure of the security, but also can extend the life of the building itself, from the fundamental to reduce the incidence of building safety accidents, and thus promote the construction Sustainable Development of Economic Construction of Construction Units. In view of this, this paper mainly analyzes the construction technology of concrete structure in construction engineering.
\end{abstract}

Key words: construction; concrete structure; construction technology

Published online: 15th July, 2017

sive construction site clean-up work, especially in the construction site of some debris and water, etc., to promptly clean up, so as not to affect the follow-up construction. Second, we must also grasp the correlation coefficient of the template. When the concrete structure is poured, the template technology is very 
critical, the location of the template, elevation, strength and storage parameters will make the concrete structure of the precision of the pouring is greatly affected, and in the pouring before the full range of familiar, Thus laying a solid foundation for the follow-up pouring links. And then the different pouring parts have to use the application of the way not to pour. In the construction project construction, the construction site of the application of the way of pouring is also different, such as in the foundation of the construction, it is necessary to use the concrete structure of the pouring material and the main structure of the concrete structure of the material to be treated differently. It is noteworthy that in the process of pouring is likely to be some problems, and the need to timely analysis of the reasons and to develop effective measures to deal with. Finally, ensure that the pouring process of uniform density. In the construction process of concrete structure of the pouring material to be analyzed, but also to ensure its compactness and uniformity, in the maximum to prevent the emergence of pouring interval situation. In order to effectively improve the effect of pouring, then you have to strictly control the concrete structure of the tamping, to ensure that the concrete structure after forming the compactness.

\subsection{Vibration of the concrete struc- ture}

The vibration of the concrete structure is the first choice for the vibrating bar and its application in the process of arrangement and use will affect the effect of vibration, in the specific use of time, not only to focus on the vibration process of strength, But also to protect the speed of vibration, in the fast-cut slowly at the same time, to vibrate a single movement of the distance control in the role of the radius of 1.5 times, the distance between the points to keep within $450 \mathrm{~mm}$. At the same time, in the vibrating time, the vibrating bar and the template, buried pipe, embedded parts and so on to maintain a certain distance, to avoid damage or displacement phenomenon, in the vibrating parts of the concrete structure Large and also accompanied by the emergence of cold seam problems, it will increase the difficulty of the construction of concrete structures, this time we have to adjust the vibrating way or speed.

\subsection{Conservation technology}

The first thing to do is to ensure the adequacy of conservation, usually to continuous conservation in $28 \mathrm{~d}$ can make the concrete structure condensation to achieve the purpose of hardening; followed by the concrete from the concrete to focus on the necessary water treatment, the application of spray Method to carry out the corresponding treatment to ensure that the concrete structure material can be a wet state; the last is in the concrete structure of the process of conservation should always pay attention to the temperature changes, to prevent the occurrence of internal and external temperature difference is too large, to timely Do the appropriate insulation and cooling treatment work. In addition, the time of curing can be set according to the thickness of the concrete structure and the corresponding construction requirements to prevent the occurrence of cracks in dry weather and cause the cost and progress of the construction project to be very seriously affected.

\section{Conclusion}

In short, in the process of construction and construction of concrete construction, concrete structure in which the role is very critical, then you need to practice in the process, slowly sum up the construction experience, and also have to carry out the corresponding research work, In the course of the study, if any problems are found, it is necessary to be resolved in a timely manner. In the concrete structure of the production, design and application of the process, the full role of their own play out to promote the development of new technologies and applications of the process, to promote the application of our national concrete structure and promotion.
[1] Tian Tai. Study on the Construction Technology of Concrete Structure in Civil Engineering Construction [J]. Heilongjiang Science and Technology Information, 2016, (19): 252.

[2] Chen Jinqiang. Construction Technology Concrete Construction Technology Research [J]. China High-tech enterprises, 2016, (01): 119-120.

[3] Liu Qian. Construction of Concrete Construction Technology Points Analysis [A]. Shijiazhuang City Han Kun Culture and Arts Co., Ltd.. 2015 Ninth essays Symposium Proceedings [C]. Shijiazhuang City Han Kun Culture and Arts Co., Ltd. 2015: 4.

\section{References}

